\title{
Chicken IgY Fc expressed by Eimeria mitis enhances the immunogenicity of $E$. mitis
}

\author{
Mei Qin ${ }^{1,2}$, Xinming Tang ${ }^{1,2}$, Guangwen Yin ${ }^{4}$, Xianyong Liu ${ }^{1,2,3}$, Jingxia Suo ${ }^{1,2}$, Geru Tao ${ }^{1,2}$, Saeed El-Ashram ${ }^{1,2}$, \\ Yuan $\mathrm{Li}^{1,2}$ and Xun SuO ${ }^{1,2,3^{*}}$
}

\begin{abstract}
Background: Eimeria species are obligate intracellular apicomplexan parasites, causing great economic losses in the poultry industry. Currently wild-and attenuated- type anticoccidial vaccines are used to control coccidiosis. However, their use in fast growing broilers is limited by vaccination side effects caused by medium and/or low immunogenic Eimeria spp. There is, therefore, a need for a vaccine with high immunogenicity for broilers.

Methods: The avian yolk sac IgY Fc is the avian counterpart of the mammalian lgG Fc, which enhances immunogenicity of Fc-fusion proteins. Here, we developed a stable transgenic Eimeria mitis expressing IgY Fc (Emi.chFc) and investigated whether the avian IgY Fc fragment enhances the immunogenicity of E. mitis. Two-week-old broilers were immunized with either Emi.chFc or wild type Eimeria and challenged with wild type E. mitis to analyze the protective properties of transgenic Emi.chFc.

Results: Chickens immunized with Emi.chFc had significantly lower oocyst output, in comparison with PBS, mock control (transgenic E. mitis expressing HA1 from H9N2 avian influenza virus) and wildtype E. mitis immunized groups after challenge, indicating that IgY Fc enhanced the immunogenicity of E. mitis.

Conclusions: Our findings suggest that IgY Fc-expressing Eimeria may be a better coccidiosis vaccine, and transgenic Eimeria expressing Fc-fused exogenous antigens may be used as a novel vaccine-delivery vehicle against a wide variety of pathogens.
\end{abstract}

Keywords: Eimeria mitis, Stable transfection, Chicken lgY Fc, Protective immune response

\section{Background}

Avian coccidiosis, an intestinal disease caused by apicomplexan parasites of the genus Eimeria, causes significant economic losses to the poultry industry throughout the world [1]. Coccidiosis can be effectively controlled by chemical pharmaceuticals. However, chemical treatment or prevention is associated with drug residues in poultry products and leads to drug resistance by the parasites [2-4]. Several Eimeria vaccines, such as Coccivac ${ }^{\circ}$, Immunocox ${ }^{\oplus}$, Paracox ${ }^{\oplus}$ and Livacox ${ }^{\oplus}$ have been used successfully for the control of coccidiosis in breeders and layers $[1,5]$. However, vaccines have not been widely used in broilers due to vaccination side effects and

\footnotetext{
* Correspondence: suoxun@cau.edu.cn

'State Key Laboratory of Agrobiotechnology, College of Veterinary Medicine,

China Agricultural University, Beijing 100193, China

${ }^{2}$ National Animal Protozoa Laboratory \& College of Veterinary Medicine,

China Agricultural University, Beijing 100193, China

Full list of author information is available at the end of the article
}

relatively low immunogenicity [6-8]. Host immunity to avian coccidiosis is complex, predominantly cellmediated and requires at least two to three times of reinfection after vaccination with medium or low immunogenic Eimeria spp. [1, 9]. There is, therefore, a need for a highly effective vaccine which can elicit enhanced immune response to Eimeria to prevent coccidiosis for broilers.

The neonatal Fc receptor ( $\mathrm{FcRn}$ ) can transport IgG antibody across mucosal surfaces [10-12] and the FcRnmediated transport was used to enhance the immunogenicity of Fc-expressing plasmid DNA and Fc-fusion proteins in mammals $[12,13]$. Previous research revealed that the avian IgY Fc receptor (FcRY) exhibits the same binding character to IgY as the mammalian FcRn [14, 15]. We hypothesize that the chicken IgY Fc fragment facilitates the uptake of Eimeira antigen leading to enhanced immune responses against Eimeria. Importantly, 
transient and stable transfection of Eimeira mitis are well established [16]. In the present study, we developed transgenic Eimeria mitis expressing IgY Fc (Emi.chFc) and showed that broilers immunized with Emi.chFc elicited higher protective immune response against wild-type E. mitis than wild type E. mitis-immunized chickens.

\section{Methods}

\section{Ethics statement}

All animal research was approved by the Beijing Association for Science and Technology (approval ID SYXK (Beijing) 2007-0023) and was in compliance with Beijing Laboratory Animal Welfare and Ethics guidelines as issued by the Beijing Administration Committee of Laboratory Animals. All animal studies were also performed in accordance with the China Agricultural University Institutional Animal Care and Use Committee guidelines (ID:SKLAB-B-2010-003) and approved by the animal welfare committee of China Agricultural University.

\section{Parasites, chickens and cell culture}

Oocysts of E. mitis (Zhuozhou strain) were propagated according to established protocols [17]. The transgenic E. mitis expressing HA1 region from $\mathrm{H} 9 \mathrm{~N} 2$ avian influenza virus (Emi.HA1) used as a mock control for IgY Fc transgenic E. mitis (see below) in the study was well established in our laboratory (unpublished data).

One-day-old Arbor Acre (AA) broiler chickens were purchased from Beijing Arbor Acres Poultry Breeding Co., Ltd. They were housed in isolators and fed with pathogen-free diet and water.

Madin-Darby bovine kidney (MDBK) cells were cultured in DMEM medium supplemented with fetal bovine serum $(10 \% \mathrm{v} / \mathrm{v})$ and $1000 \mathrm{U}$ penicillin/streptomycin in a humidified atmosphere with $5 \% \mathrm{CO}_{2}$ at $37^{\circ} \mathrm{C}$.

\section{Plasmid construction and transfection of $E$. mitis}

Chicken IgY Fc fragment containing the $\mathrm{CH} 2, \mathrm{CH} 3$ and CH4 domains (GenBank: X07174) was synthesized by Aoke Peak Biological Science and Technology Co.Ltd. (China, Beijing) after codon optimization.

The double expression-cassette plasmid, Mic-DHFREYFP/ACT-chFc-ACT (pMDEAAsschFcA) was constructed based on the pMic-EYFP/ACT-RFP plasmid as previously reported [18], with the RFP and EYFP genes replaced by chFc (Primer P1/P2) and TgDHFR-EYFP (Primer P3/P4) genes, respectively (Fig. 1a and Table 1). With the help of the integrated pyrimethamine resistance gene DHFR-TSm2m3, highly effective fluorescent oocysts were obtained [16, 19]. The plasmid DNA was

a
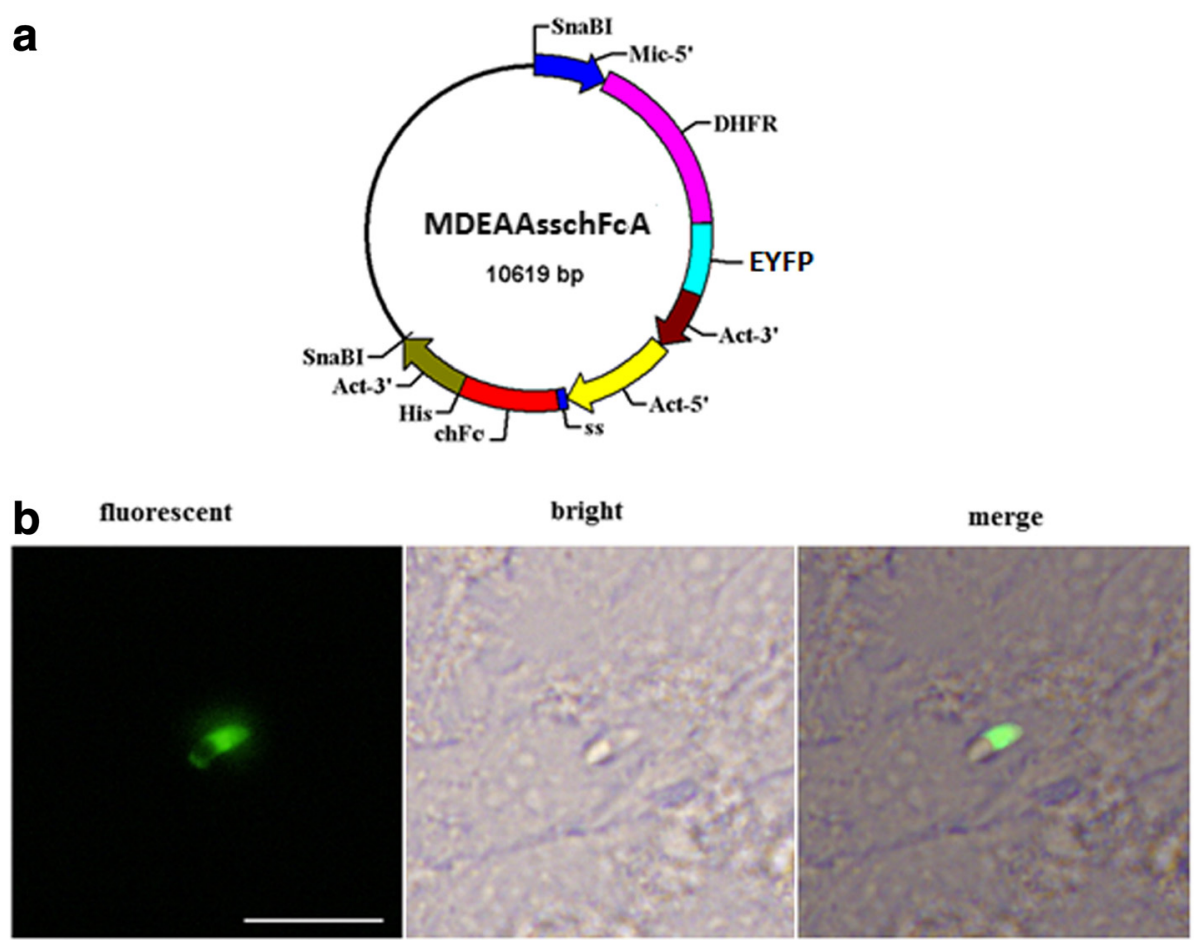

Fig. 1 Schematic representation of EYFP containing plasmid and transient expression after inoculation in MDBK cells. a Expression cassettes are colored. The EYFP coding region is flanked by the Mic2 promoter and 3'region of actin from E.tenella. The foreign protein region is flanked by the promoter of actin (Act), 3'region of actin and signal sequence ( $\mathrm{ss}, 85 \mathrm{bp}$ ) derived from dense granule protein 8 (GRA8) of Toxoplasma gondii. b Sporozoites transfected with theMDEAAsschFcA plasmid and grown in MDBK cells. Scale-bar: $20 \mu \mathrm{m}$ 
Table 1 Primers used in PCR

\begin{tabular}{|c|c|c|}
\hline Primer & Forward primer & Reverse primer \\
\hline \multirow[t]{2}{*}{$\mathrm{P} 1 / \mathrm{P} 2$} & $5^{\prime}$ CCTAGGCAGGCAGGCAAGCCTAGGGC & 5'CCGCGGTAGTGGTGGTGGTGG \\
\hline & TGCGGGAACAGCCAGT3' & TGGTGCGCGACACAAG3' \\
\hline \multirow[t]{2}{*}{ P3/P4 } & 5'GGTACCATGCAGAAGCCGGTGTGT & 5'CCTAGGAAGCTTCTTGTACAGCT \\
\hline & CTGGT3' & CGTCC3' \\
\hline P5/P6 & 5'ATGGTGAGCAAGGGCGAGGA3' & 5'AAGCTTCTTGTACAGCTCGT3' \\
\hline SP1 & - & 5'AAGGCGAGCGAAGCTGTTCACT3' \\
\hline SP2 & - & 5'CCATGCTTGGAGGAAACTTTGC3' \\
\hline SP3 & _ & 5'GCCTCTCGAAGGATCTGAATGC3' \\
\hline
\end{tabular}

linearized by SnaBI restriction enzyme to release the two expression cassettes from the backbone of the plasmid.

For plasmid transfection, $10^{7}$ sporozoites freshly purified through a DE-52 cellulose column, $10 \mu \mathrm{g}$ linearized DNA plasmid and $5 \mu \mathrm{l}$ SnaBI were subjected to Nucleofector transfection (Program U-033, AMAXA, Switzerland). Half of the transfected sporozoites were examined for transient EYFP expression after inoculation onto confluent MDBK cells. The other half of the transfected sporozoites were inoculated into 7-day-old chickens via the cloacal route. The oocysts in fecal samples at 5-8 days post-inoculation were collected, purified and examined by fluorescence microscopy (Leica, Germany) [18, 20].

The transfected oocysts expressing EYFP (Emi.chFc) were selected in chickens by pyrimethamine (Sigma-Aldrich Co., St. Louis, Mo., USA) supplemented in feed and the $\mathrm{MoFlo}^{\circ}$ Cell Sorter (Dako-Cytomation, Fort Collins, CO) on the singlecell mode, and then inoculated into coccidian-free chickens for the propagation of next generation [20]. The purified oocysts were re-suspended in $2.5 \%$ $\mathrm{KCr}_{2} \mathrm{O}_{7}$ and stored at $4{ }^{\circ} \mathrm{C}$ for further experiments.

\section{Genomic DNA analysis of transgenic $E$. mitis}

Genomic DNA from Emi.chFc was prepared as previously described [21]. PCR primer pairs (forward and reverse primer sequences) targeting the chFc and YFP genes were $\mathrm{P} 1 / \mathrm{P} 2$ and P5/P6, respectively (Table 1 ). The chicken Fc and YFP genes were amplified employing the above total genomic DNA as templates. No template and mocktransfected parasite DNA samples were served as negative controls and plasmid MDEAAsschFcA was used as a positive control.

A genome walking kit (Takara, Dalian, China) was exploited to detect whether the plasmid was integrated into the E. mitis genome. According to the sequence of the MIC2 promoter, specific reverse primers were designed, SP1, SP2 and SP3 (Table 1). The forward primers AP1, AP2, AP3 and AP4 were supplied in the Genome Walking Kit. The following PCR amplification conditions were used: First-round PCR reaction: $94{ }^{\circ} \mathrm{C}$ for
$1 \mathrm{~min}, 98^{\circ} \mathrm{C}$ for $1 \mathrm{~min}, 94^{\circ} \mathrm{C}$ for $30 \mathrm{~s}, 65^{\circ} \mathrm{C}$ for $1 \mathrm{~min}$, and $72{ }^{\circ} \mathrm{C}$ for $2 \mathrm{~min}$ for 5 cycles; $94{ }^{\circ} \mathrm{C}$ for $30 \mathrm{~s}, 25^{\circ} \mathrm{C}$ for $3 \mathrm{~min}$, and $72{ }^{\circ} \mathrm{C}$ for $2 \mathrm{~min} ; 94{ }^{\circ} \mathrm{C}$ for $30 \mathrm{~s}, 65^{\circ} \mathrm{C}$ for $1 \mathrm{~min}$, and $72{ }^{\circ} \mathrm{C}$ for $2 \mathrm{~min} ; 94^{\circ} \mathrm{C}$ for $30 \mathrm{~s}, 65^{\circ} \mathrm{C}$ for $1 \mathrm{~min}$, and $72{ }^{\circ} \mathrm{C}$ for $2 \mathrm{~min}$ for 15 cycles; $94{ }^{\circ} \mathrm{C}$ for $30 \mathrm{~s}$, $44{ }^{\circ} \mathrm{C}$ for $1 \mathrm{~min}, 72{ }^{\circ} \mathrm{C}$ for $2 \mathrm{~min}$, and $72{ }^{\circ} \mathrm{C}$ for $10 \mathrm{~min}$. The second- and third-PCR reactions consisted of $94^{\circ} \mathrm{C}$ for $30 \mathrm{~s}, 65{ }^{\circ} \mathrm{C}$ for $1 \mathrm{~min}$, and $72{ }^{\circ} \mathrm{C}$ for $2 \mathrm{~min}$; $94{ }^{\circ} \mathrm{C}$ for $30 \mathrm{~s}, 65{ }^{\circ} \mathrm{C}$ for $1 \mathrm{~min}$, and $72{ }^{\circ} \mathrm{C}$ for $2 \mathrm{~min} ; 94{ }^{\circ} \mathrm{C}$ for $30 \mathrm{~s}, 44{ }^{\circ} \mathrm{C}$ for $1 \mathrm{~min}$, and $72{ }^{\circ} \mathrm{C}$ for $2 \mathrm{~min}$ for 15 cycles; and an extension at $72{ }^{\circ} \mathrm{C}$ for $10 \mathrm{~min}$. The third-round PCR products were selected, purified and cloned into pEASY-T1-simple vector (TransGen Biotech, China) and confirmed by DNA sequencing. The resulting sequences were then analyzed by DNAStar7.0 software, and the integration sites in the genome were identified by performing a BLAST search in the $E$. mitis DB database.

\section{Western blot analysis}

Protein extraction from Emi.chFc was carried out as previously described [21]. The total soluble protein from transgenic E. mitis was resolved by sodium dodecyl sulfate-polyacrylamide gel electrophoresis (SDS-PAGE) and electro-transferred onto a polyvinylidene difluoride (PVDF) membrane. For the detection of the target chFc protein, the membrane was probed with HRPconjugated goat anti-chicken IgY Fc directly as primary antibodies.

\section{Indirect immunofluorescence assay}

The indirect immunofluorescence assay was used to localize the foreign chicken Fc protein in E. mitis. Sporozoites purified by DE-52 anion-exchange were applied onto a poly-L-lysine-coated slide, followed by incubation in acetone at $-20{ }^{\circ} \mathrm{C}$ for $20 \mathrm{~min}$. After washing 3 times with PBS, the samples were permeabilized by incubation with Triton X-100 (0.1 \% in PBS) for 15 min. To prevent unspecific binding of the detection antibody, the samples were blocked with $1 \%$ BSA in PBS for 60 min. This was followed by $60 \mathrm{~min}$ incubation with $\mathrm{Cy3}$-conjuated goat anti-chicken IgY Fc fragment (Jackson). The transgenic 
Table 2 Transfection efficiency of transgenic E. mitis following nucleofector transfection and selection in chickens

\begin{tabular}{cccccc}
\hline & \multicolumn{6}{c}{ Percentage of fluorescent oocysts in total shed oocysts } \\
\cline { 2 - 6 } & 1 1st & 2nd & 3rd & 4 th & 5 th \\
\hline Emi.chFc & $0.2 \%$ & $30 \%$ & $80 \%$ & $91 \%$ & $93 \%$ \\
\hline
\end{tabular}

E. mitis sporozoites were examined under a laser scanning confocal microscope (SP5, Leica, Germany).

\section{Immunogenicity assay}

Two-week-old AA broilers were orally vaccinated with 1000 freshly sporulated Emi.chFc, 1000 freshly sporulated wild-type (WT) oocysts of E. mitis, or 1000 freshly sporulated Emi.HA1 (as the mock control) $(n=6)$. An unvaccinated control group was orally given PBS. Fourteen days after vaccination, all groups were challenged with $4 \times 10^{5}$ wild type sporulated oocysts of $E$. mitis. Oocysts shedding per bird was determined 5-8 d after the vaccination and challenge infection using the McMaster egg counting chamber $[21,22]$.

\section{Statistical analysis}

Statistical analysis was performed using one-way ANOVA in the IBM SPSS Statistics 20 for Windows software. Differences between groups with a $P$-value of $<0.05$ were considered to be statistically significant.

\section{Results}

chFc - E. mitis transfection and YFP expression

E. mitis sporozoites were transfected with the plasmid MDEAAsschFcA expressing YFP protein. EYFP expression was observed in transgenic sporozoites (Fig. 1b).

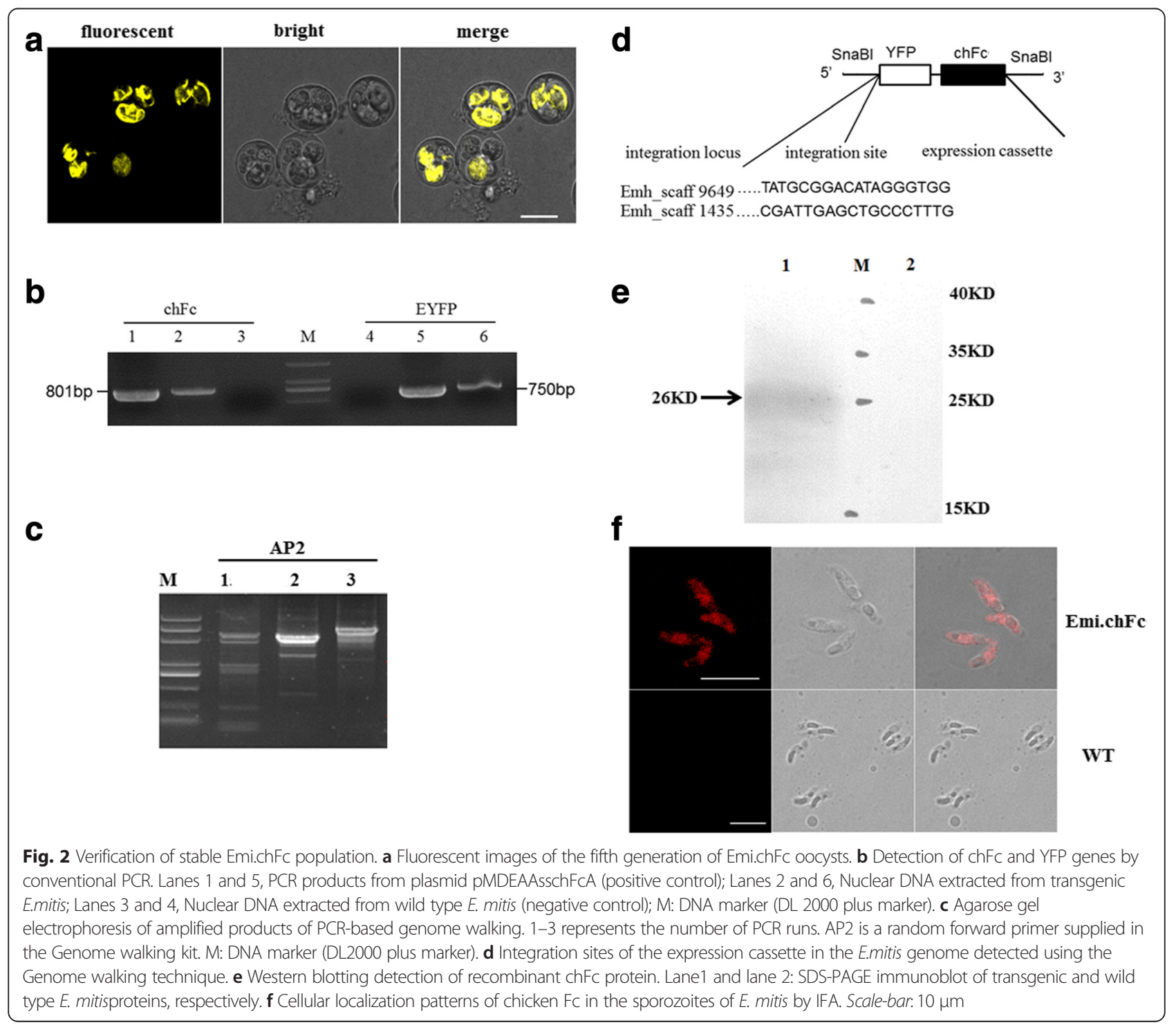


Pyrimethamine-resistant transgenic E. mitis was selected by adding $150 \mu \mathrm{g} / \mathrm{mg}$ pyrimethamine to the host, chicken diet. In the first generation, $0.2 \%$ of the excreted oocysts expressed the YFP protein (Table 2). Subsequent passages were selected for both pyrimethamine resistance and YFP expression using pyrimethamine-containing diet and FluorescenceActivated Cell-Sorting (FACS) of oocysts. Consequently, there has been a gradual rise in the proportion of YFP expressing oocysts in each generation up to the fifth passage (Fig. 2a), where the fluorescenceexpressing population reached $93 \%$ (Table 2).

\section{Validation of stable transfection of $E$. mitis}

PCR detection of YFP and chFc genes yielded products of the expected size using the genomic DNA of sporulated oocysts of transgenic $E$. mitis as the template (Fig. 2b).

The integration sites of the transfected plasmid into the $E$. mitis nuclear genome were identified by the genome walking assay from the nuclear genome. As shown in Fig. 2c and d, sequence analysis showed that the construct was integrated into the genome of E. mitis, with two integration sites (one in Emh_scaff1435 and the other in Emh_scaff9649). As the sites with the inserted integration have not been annotated yet, we cannot clarify the location of plasmid integration.

SDS-PAGE resolving gel of the recombinant chicken Fc protein of transgenic E. mitis was $26 \mathrm{KDa}$, indicating the expression of chFc protein in the transgenic E. mitis (Fig. 2e). IFA showed that chicken Fc protein was expressed in the whole sporozoites (Fig. 2f).
Chicken IgY Fc delivered by $E$. mitis enhances protection against wild type $E$. mitis challenge infection

Chickens were immunized by a single inoculation of the transgenic oocysts and then challenged with the wild type parasites. Oocysts excreted 5-8 days after vaccination in the Emi.chFc group $\left(1.2 \times 10^{7} / \mathrm{bird}\right)$ was significantly lower than the WT group $\left(4.7 \times 10^{7} /\right.$ bird $)(P<0.05)$. Two weeks after the vaccination, each bird in the Emi.chFc, WT and PBS groups was challenged with $4 \times 10^{5} \mathrm{WT}$ E. mitis. There was a significant reduction by 85 and $78 \%$, respectively, in oocysts shedding 5-8 days after the challenge in the Emi.chFc group $\left(9.2 \times 10^{4} /\right.$ bird $)$ in comparison with the WT group $\left(6.0 \times 10^{5} /\right.$ bird $)(P<0.01)$ and the Emi.HA1 control group $\left(4.0 \times 10^{5} /\right.$ bird $)$ while the oocysts output in the PBS group after the challenge was $5.0 \times 107 /$ bird (Fig. 3). The observed decline of oocyst output for birds vaccinated with Emi.chFc suggested that chicken IgY Fc delivered by $E$. mitis could enhance immunogenicity of the parasite and consequently confer better protection against $E$. mitis infection.

\section{Discussion}

We showed that transgenic E. mitis expressing chicken IgY Fc fragment provoked better protective immune response than the parental $E$. mitis against $E$. mitis infection. Chickens immunized with E. mitis-vectored chicken IgY Fc fragment significantly reduced oocysts output in comparison with control groups immunized with the parental E. mitis, transgenic E. mitis expressing HA1 or PBS after challenge infection.

The mechanisms of enhanced protective immunogenicity by expressing isolated chicken IgY Fc in E. mitis are unknown, but the enhanced immunogenicity could be attributed to the interaction between chicken IgY Fc and its

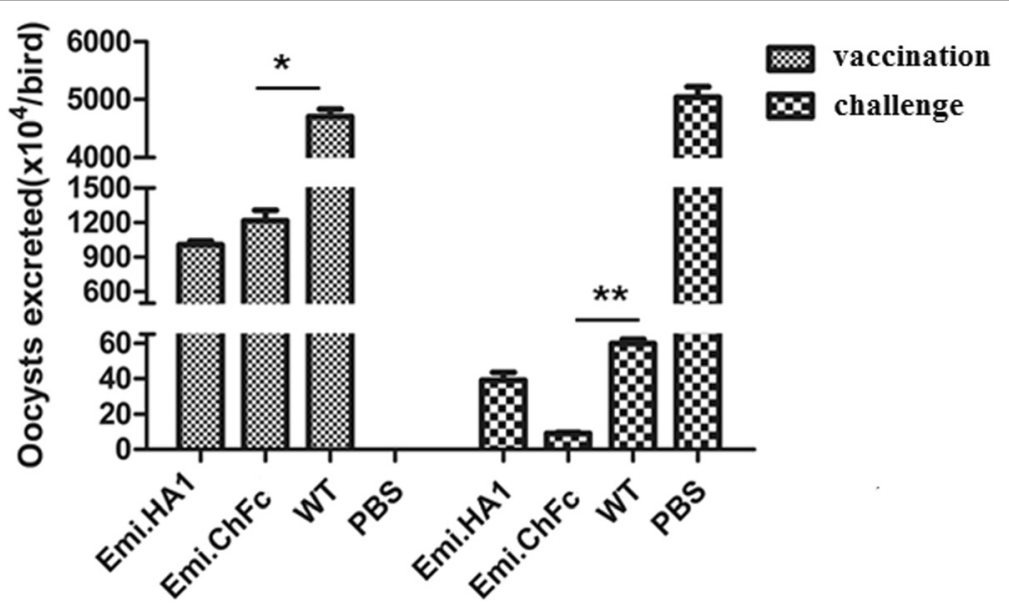

Fig. 3 Oocysts excretion after vaccination and challenge infection in chickens. Two-week-old AA broiler chickens $(n=6)$ were vaccinated with 1000 Emi.chFc, Emi.HA1 or WT oocysts or PBS and challenged with $4 \times 10^{5}$ WT oocysts two weeks after vaccination. Feces from each group were collected 5-8 days post-vaccination and challenge. Oocysts shedding per gram of feces was determined using a McMaster egg counting chamber. The data was pooled of four independent experiments with similar results and were expressed as the mean \pm SD 
receptors which stimulated a micro-environment enhancing antigen intake. Fc receptors are key players of the immune system and link the acquired humoral immune response with innate cellular effector responses [23]. It has been postulated that mammals Fc-mediated effector function plays an important role in protective immunity to HIV in humans [24]. The avian yolk sac IgY receptor ( $F c R Y)$ is the mammalian phospholipase A2 receptor orthologue and the functional counterpart of mammalian FcRn. FcRY was shown to facilitate bidirectional transcytosis and recycling of chicken IgY, suggesting it may regulate homeostasis of IgY [25]. Chicken FcRY might facilitate $E$ mitis antigen presentation and thus the protective immune response of chickens. Another chicken IgY Fc receptor is CHIRAB1, which has unique high-affinity to chicken $F_{C}[24,26]$ and is mainly expressed on immature and mature B lymphocytes, and cells of the innate immune system such as monocytes, macrophages and NK cells [23]. The binding of expressed Fc to CHIRAB1 may also contribute to the enhanced immune response in chickens immunized with the chicken Fc expressing oocysts. The exact role and mechanisms of chicken IgY Fc in boosting host immunity against infections are yet to be explored.

Although live parasites vaccine for preventing coccidiosis are very successful in breeder and layer chickens, they are less justifiable economically for short-lived broilers due to the vaccination side effects and the need for repeated vaccinations with medium or low immunogenic Eimeria spp.. Our findings suggest that chicken Fc expressed by $E$. mitis have the potential to enhance immune response to coccidian antigens and transgenic $E$. mitis delivering chicken IgY Fc fragment could be used as an alternative, more effective coccidiosis vaccine. Further research regarding the development of transgenic $E$. mitis or $E$. tenella as a novel vaccine vector expressing chicken IgY Fc-fused exogenous antigens would be worthwhile.

\section{Conclusions}

Transgenic E. mitis expressing chicken IgY Fc fragment enhanced better protective immune response against $E$. mitis. Transgenic Eimeria expressing Fc-fused exogenous antigens may be used as a novel vaccine-delivery vehicle against a wide variety of pathogens.

\section{Competing interests}

All authors declare that they have no competing interests.

\section{Authors' contributions}

$M Q, X Y L$ and XS conceived and designed the project, participated in general data analysis and in writing the manuscript. JXS performed protein

identification and genome walking. XMT, GWY, SEA, GRT and YL did animal experiments and wrote the manuscript. All authors read and approved the final manuscript.

\section{Acknowledgments}

This study was supported by the National Natural Science Foundation of China (Key project, 31330076; 31001060) and Research and Innovation Project for College Graduates of China Agricultural University (2013YJ005).

\section{Author details}

'State Key Laboratory of Agrobiotechnology, College of Veterinary Medicine, China Agricultural University, Beijing 100193, China. ${ }^{2}$ National Animal Protozoa Laboratory \& College of Veterinary Medicine, China Agricultural University, Beijing 100193, China. ${ }^{3}$ Key Laboratory of Animal Epidemiology and Zoonosis of Ministry of Agriculture, China Agricultural University, Beijing 100193, China. ${ }^{4}$ Engineering Laboratory of Animal Pharmaceuticals, College of Animal Science, Fujian Agriculture and Forestry University, Fuzhou, Fujian Province 350002, China.

Received: 12 June 2015 Accepted: 14 March 2016

Published online: 21 March 2016

\section{References}

1. Shirley MW, Smith AL, Tomley FM. The biology of avian Eimeria with an emphasis on their control by vaccination. Adv Parasitol. 2005;60:285-330.

2. Peek HW, Landman WJ. Higher incidence of Eimeria spp. field isolates sensitive for diclazuril and monensin associated with the use of live coccidiosis vaccination with paracox-5 in broiler farms. Avian Dis. 2006;50(3):434-9.

3. Michels MG, Bertolini LC, Esteves AF, Moreira P, Franca SC. Anticoccidial effects of coumestans from Eclipta alba for sustainable control of Eimeria tenella parasitosis in poultry production. Vet Parasitol. 2011;177(1-2):55-60.

4. Dalloul RA, Lillehoj HS. Poultry coccidiosis: recent advancements in control measures and vaccine development. Expert Rev Vaccines. 2006;5(1):143-63.

5. Sharman PA, Smith NC, Wallach MG, Katrib M. Chasing the golden egg: vaccination against poultry coccidiosis. Parasite Immunol. 2010;32(8):590-8.

6. Williams RB. Epidemiological aspects of the use of live anticoccidial vaccines for chickens. Int J Parasitol. 1998;28(7):1089-98.

7. Chapman HD, Cherry TE, Danforth HD, Richards G, Shirley MW, Williams RB. Sustainable coccidiosis control in poultry production: the role of live vaccines. Int J Parasitol. 2002;32(5):617-29.

8. Danforth HD. Use of live oocyst vaccines in the control of avian coccidiosis: experimental studies and field trials. Int J Parasitol. 1998;28(7):1099-109.

9. Blake DP, Tomley FM. Securing poultry production from the ever-present Eimeria challenge. Trends Parasitol. 2014;30(1):12-9.

10. Dickinson BL, Badizadegan K, Wu Z, Ahouse JC, Zhu X, Simister NE, et al. Bidirectional FCRn-dependent lgG transport in a polarized human intestinal epithelial cell line. J Clin Invest. 1999;104(7):903-11.

11. Roopenian DC, Akilesh S. FCRn: the neonatal Fc receptor comes of age. Nat Rev Immunol. 2007;7(9):715-25.

12. Yoshida M, Kobayashi K, Kuo TT, Bry L, Glickman JN, Claypool SM, et al. Neonatal Fc receptor for lgG regulates mucosal immune responses to luminal bacteria. J Clin Invest. 2006;116(8):2142-51.

13. Ye L, Zeng R, Bai Y, Roopenian DC, Zhu X. Efficient mucosal vaccination mediated by the neonatal Fc receptor. Nat Biotechnol. 2011;29(2):158-63.

14. Linden CD, Roth TF. IgG receptors on foetal chick yolk sac. J Cell Sci. 1978; 33:317-28.

15. Tressler RL, Roth TF. IgG receptors on the embryonic chick yolk sac. J Biol Chem. 1987;262(32):15406-12.

16. Qin M, Liu XY, Tang XM, Suo JX, Tao GR, Suo X. Transfection of Eimeira mitis yellow fluorescent protein as reporter and the endogenous development of the transgenic parasite. PLoS One. 2014;9(12):e114188.

17. Long PL, Millard B, Joyner L, Norton CC. A guide to laboratory techniques used in the study and diagnosis of avian coccidiosis. Folia Vet Lat. 1975;6(3): 201-17.

18. Yin G, Liu X, Zou J, Huang X, Suo X. Co-expression of reporter genes in the widespread pathogen Eimeria tenella using a double-cassette expression vector strategy. Int J Parasitol. 2011;41(8):813-6.

19. Hanig S, Emtzeroth R, Kurth M. Chimeric fluorescent reporter as a tool for generation of transgenic Eimeria (Apicomplexa, Coccidia) strains with stage specific reporter gene expression. Parasitol Int. 2012;61(3):391-8.

20. Yan W, Liu X, Shi T, Hao L, Tomley FM, Suo X. Stable transfection of Eimeria tenella: constitutive expression of the YFP-YFP molecule throughout the life cycle. Int J Parasitol. 2009;39(1):109-17. 
21. Liu X, Zou J, Yin G, Su H, Huang X, Li J, et al. Development of transgenic lines of Eimeria tenella expressing Mze-enhanced yellow fluorescent protein (M2e-EYFP). Vet Parasitol. 2013;193(1):1-7.

22. Huang $X$, Zou J, Xu H, Ding Y, Yin G, Liu X, et al. Transgenic Eimeria tenella expressing enhanced yellow fluorescent protein targeted to different cellular compartments stimulated dichotomic immune responses in chickens. J Immunol. 2011;187(7):3595-602.

23. Viertlboeck BC, Schweinsberg S, Hanczaruk MA, Schmitt R, Du Pasquier L, Herberg FW, et al. The chicken leukocyte receptor complex encodes a primordial, activating, high-affinity IgY Fc receptor. Proc Natl Acad Sci U S A. 2007;104(28):11718-23.

24. Lewis GK. Role of Fc-mediated antibody function in protective immunity against HIV-1. Immunology. 2014;142(1):46-57.

25. Tesar DB, Cheung EJ, Bjorkman PJ. The chicken yolk sac IgY receptor, a mammalian mannose receptor family member, transcytoses lgY across polarized epithelial cells. Mol Biol Cell. 2008;19(4):1587-93.

26. Taylor Al, Beavil RL, Sutton BJ, Calvert RA. A monomeric chicken IgY receptor binds IgY with 2:1 stoichiometry. J Biol Chem. 2009;284(36): 24168-75.

Submit your next manuscript to BioMed Central and we will help you at every step:

- We accept pre-submission inquiries

- Our selector tool helps you to find the most relevant journal

- We provide round the clock customer support

- Convenient online submission

- Thorough peer review

- Inclusion in PubMed and all major indexing services

- Maximum visibility for your research

Submit your manuscript at www.biomedcentral.com/submit
Biomed Central 